\title{
The cost trajectory of the diagnostic care pathway for children with suspected genetic disorders
}

\author{
Nick Dragojlovic, PhD ${ }^{1}{ }^{1}$, Clara D. M. van Karnebeek, MD, PhD ${ }^{2,3,4}$, Aisha Ghani, BA ${ }^{3}$, \\ Dallas Genereaux, MPH, BSc ${ }^{5}$, Ellen Kim, BSc (Pharmacy) ${ }^{1}$, Patricia Birch, MSc, RN ${ }^{5}$, CAUSES Study, \\ Alison M. Elliott, $\mathrm{PhD}^{3,5}$, Jan M. Friedman, $\mathrm{MD}, \mathrm{PhD}^{5}$ and Larry $\mathrm{D}$. Lynd, $\mathrm{PhD}^{1,6}$
}

Purpose: This study describes the cost trajectory of the standard diagnostic care pathway for children with suspected genetic disorders in British Columbia, Canada.

Methods: Average annual per-patient costs were estimated using medical records review and a caregiver survey for a cohort of 498 children referred to BC Children's and Women's Hospitals (C\&W) with unexplained intellectual disability (the TIDE-BC study) and families enrolled in the CAUSES study, which offered diagnostic genome-wide sequencing (GWS; exome and genome sequencing) to 500 families of children with suspected genetic disorders.

Results: Direct costs peaked in the first year of patients' diagnostic odyssey, with an average of C $\$ 2257$ per patient (95\% confidence interval [CI] C \$2074, C\$2441) for diagnostic testing and C\$631 (95\% CI C $\$ 543$, C \$727) for specialist consultations at C\&W. In subsequent years, direct costs accrued at a constant rate, with an estimated annual per-patient cost of C\$511 (95\% CI C\$473, C\$551) for diagnostic testing and C $\$ 334$ (95\% CI C $\$ 295, \mathrm{C} \$ 369)$ for consultations at C\&W. Travel costs and caregiver productivity loss associated with attending diagnosis-related physician appointments averaged C\$1907/family/year.

Conclusions: The continuing long-term accrual of costs by undiagnosed patients suggests that economic evaluations of diagnostic GWS services should use longer time horizons than have typically been used.

Genetics in Medicine (2020) 22:292-300; https://doi.org/10.1038/s41436019-0635-6

Keywords: diagnostic pathway; diagnostic costs; cost trajectory; genetic disorders; genome-wide sequencing

\section{INTRODUCTION}

Diagnostic genome-wide sequencing (GWS; exome or genome sequencing) is increasingly being used to obtain etiologic diagnoses for patients suspected of having a genetic disorder. ${ }^{1-3}$ This has contributed to a rapid increase in the diagnostic rate for children with developmental disorders and rare diseases. ${ }^{3,4}$ However, GWS testing is costly; for example, the typical cost per patient for exome sequencing (ES) is estimated at about US $\$ 2000$ for singleton ES and about US $\$ 5000$ for trio ES. ${ }^{5}$ Nevertheless, many recent economic evaluations of ES as a diagnostic test for children with suspected genetic disorders have found that using ES as a first-line test may be cost-saving because the cost of ES can be offset by the cost of averted diagnostic care for patients who receive an early diagnosis through ES. ${ }^{6}$

The generalizability of these studies is, however, limited by a number of factors. First, there is substantial variability in published reports of the diagnostic yield of $\mathrm{ES}^{2,5,6}$ the cost of delivering the test, ${ }^{5,6}$ and the cost of the standard diagnostic pathway (which includes non-GWS diagnostic tests, physician consultations focused on obtaining a diagnosis, and related services like genetic counseling) for heterogeneous cohorts of pediatric patients with suspected genetic disorders (Table S1) ${ }^{7-14}$ Indeed, reported per-patient costs for standard care range from about US $\$ 3300^{11}$ to US\$ $\$ 19,100^{10}$ per patient. This variability may be partly driven by small sample sizes ${ }^{6}$ (all but two studies ${ }^{9,13}$ in Table S1 involved fewer than 100 patients), but also likely reflects different periods during which patients' health-care utilization was observed (ranging from $1.1^{11}$ to $6.6^{7}$ years); different health-care systems (Australia, ${ }^{11,12,14}$ the Netherlands, ${ }^{7,8,13}$ and the United States $\left.{ }^{9,10}\right)$; and different patient populations including children with neurological or neurodevelopmental conditions, ${ }^{7,8,10,13}$ patients with suspected Mendelian disorders, ${ }^{11,12,14}$ and unselected patients seen in a medical genetics clinic. ${ }^{9}$

${ }^{1}$ Collaboration for Outcomes Research and Evaluation, Faculty of Pharmaceutical Sciences, University of British Columbia, Vancouver, BC, Canada; ${ }^{2}$ Department of Pediatrics, Centre for Molecular Medicine and Therapeutics, University of British Columbia, Vancouver, BC, Canada; ${ }^{3}$ BC Children's Hospital Research Institute, Vancouver, BC, Canada; ${ }^{4}$ Departments of Pediatrics and Clinical Genetics, Emma Children's Hospital, Academic University Medical Centres, Amsterdam, The Netherlands; ${ }^{5}$ Department of Medical Genetics, Faculty of Medicine, University of British Columbia, Vancouver, BC, Canada; ${ }^{6}$ Centre for Health Evaluation and Outcomes Sciences, Providence Health Research Institute, Vancouver, BC, Canada. Correspondence: Larry D. Lynd (larry.lynd@ubc.ca) 
Second, and relatedly, the patients studied in most of these investigations are unlikely to be representative of the broader patient population with suspected genetic disorders because the authors tend to report costs and outcomes either for patients who received diagnostic GWS after exhausting standard diagnostic options ${ }^{7,10}$ or for highly selected cohorts with strong indications of a Mendelian disorder. ${ }^{11,12,14}$ This limits the confidence with which these results can be used in economic evaluations of GWS as a first-line test for the broader patient population with etiologically heterogeneous conditions like intellectual disability, since patients who are diagnosed by conventional means without using GWS are likely to be systematically different from those who remain undiagnosed for long periods. While two studies have reported the average cost of standard diagnostic evaluations for larger, unselected cohorts presenting at a general genetics $\operatorname{clinic}^{9}(N=461)$ and a pediatric neurology $\operatorname{clinic}^{13}(N=150)$, neither study examined the real-world trajectory (i.e., the timing) of diagnosis-related costs accrued by these patients in detail, which is a crucial consideration when evaluating the potential cost-effectiveness of first-line GWS.

Finally, because GWS is typically offered through specialist clinics (e.g., medical genetics, pediatric neurology, etc.), costing of diagnostic tests and consultations is generally limited to costs accrued in a given tertiary care center. However, for undiagnosed patients, it is likely that substantial costs related to a patient's quest for a diagnosis accrue via ongoing visits to their primary care provider and/or community-based pediatricians and other specialists. Thus, the objective of this study was to comprehensively describe the cost trajectory of the standard diagnostic pathway for a broadly representative group of patients with suspected genetic disorders. By leveraging data on more than 700 patients, reporting on annual average per-patient costs, and providing data on physician visits in the community and families' travel and time costs, this study makes an important and novel contribution to the body of evidence on the cost of the diagnostic pathway for patients with suspected genetic disorders.

\section{MATERIALS AND METHODS}

The present study explores the accumulation of diagnosisrelated health-care utilization costs over time in two cohorts of children with suspected genetic disorders who participated in research studies at BC Children's and Women's Hospitals $(\mathrm{C} \& W)$ in Vancouver, British Columbia (BC), Canada. These cohorts included patients who ultimately received GWS because they were part of the overall patient population of children with suspected genetic disorders and must be included in any study that seeks to be representative. However, because the vast majority of diagnostic tests and all the physician consultations recorded were conducted prior to patients receiving GWS, our data also reflect the standard (pre-GWS) diagnostic pathway for this patient population. Specifically, we focus on three types of costs: (1) the direct costs of diagnostic testing to the health-care system (including laboratory testing and diagnostic imaging), (2) the direct cost to the health-care system of physician appointments that were focused on obtaining an etiologic diagnosis for the patient's condition, and (3) the travel and time costs incurred by caregivers when accompanying a child to diagnosis-specific medical visits. Unless otherwise noted, all costs are reported in 2016 Canadian dollars. Ethics approval for both studies was obtained from the Children's and Women's Research Ethics Board (University of British Columbia and C\&W), and informed consent was obtained from all participating families.

\section{The TIDE-BC study: diagnostic tests}

The Treatable Intellectual Disability Endeavor in British Columbia (TIDE-BC) study tracked a cohort of 498 pediatric patients with unexplained intellectual disability referred to C\&W for assessment between 2011 and 2014, and delivered an innovative diagnostic protocol, including a set of biochemical and genetic tests designed to identify treatable inborn errors of metabolism. ${ }^{15,16}$ Twenty percent (101/498) of these patients ultimately received exome or genome sequencing, ${ }^{17}$ with approximately 60 patients receiving GWS through the CAUSES study described below. Members of the research team reviewed patients' C\&W charts to identify all diagnostic tests received by patients, which were then classified into five major categories: biochemical and molecular genetic tests, cytogenetic tests, diagnostic imaging, and physiological tests like electrocardiograms (ECG) (Table 1). A conservative approach was taken to classifying a test as diagnostic such that only one instance of most tests was included in the data set (the exceptions being the biochemical screening tests in the TIDE protocol's first tier $^{15,16}$ and neuroimaging tests, including magnetic resonance imaging, computed tomography scans, and ultrasound exams of the brain). Test information was entered into a REDCap database ${ }^{18}$ along with the date on which the test was ordered. Unit costs were calculated for each type of test included in the database as well as the costs of collecting patient samples for testing, following Canadian guidelines ${ }^{19}$ (see Tables S2 to S6). In addition, mean values and 95\% confidence intervals (CIs) for all costs measured at the patient level were estimated using bootstrap resampling (1000 samples with the same number of elements as the original sample drawn with replacement from each cost distribution).

To explore patients' cost trajectories, the start date of a patient's diagnostic odyssey (the "baseline") was defined as the date on which the first diagnostic test captured in the database was ordered. The year of the diagnostic odyssey in which each test in the database was performed was calculated as the number of years elapsed between the baseline date and the test date rounded up to the nearest whole year. The total cost of testing incurred by each patient in each year (excluding the cost of GWS testing) was calculated, and the average per-patient diagnostic testing cost in each year of the diagnostic odyssey was calculated by dividing the total cost of all tests performed in a given year for patients who were undiagnosed at the beginning of that year by the number of these patients. 

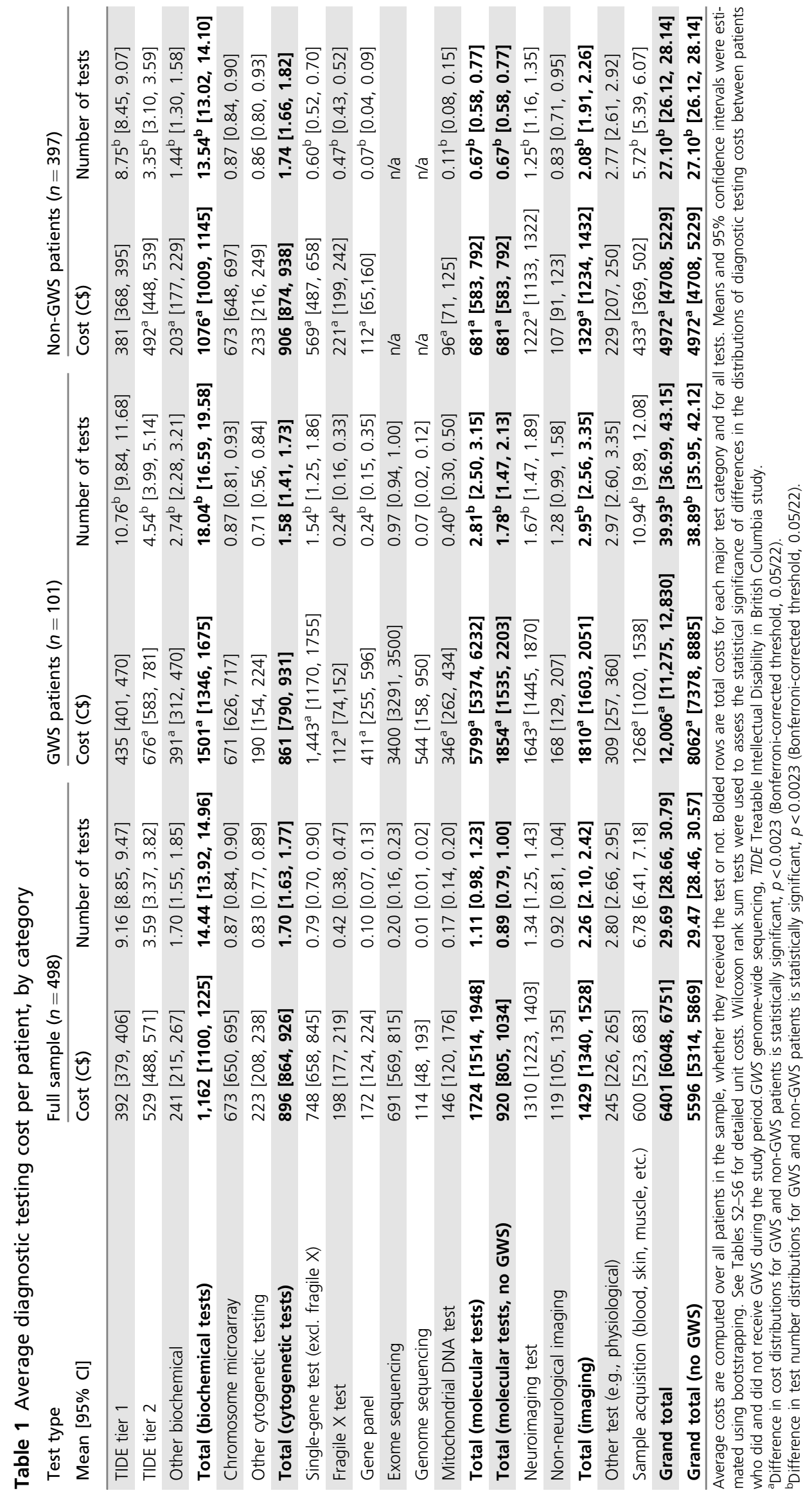


\section{The CAUSES study}

The Clinical Assessment of the Utility of Sequencing and Evaluation as a Service (CAUSES) study piloted a proposed service delivery model for GWS in British Columbia in a translational clinical research environment between 2015 and 2018 and offered exome or genome sequencing to 530 children with suspected genetic disorders from 500 families and their parents, in an effort to obtain a genetic diagnosis for the children's conditions. ${ }^{5,20}$

\section{Physician consultations at C\&W}

Retrospective data on outpatient physician consultations at $\mathrm{C} \& \mathrm{~W}$, including the date of the visit and the health-care provider, were manually abstracted from 91 CAUSES patients' medical records using the electronic medical record (EMR) system at C\&W by a principal coder (E.K.) and selectively reviewed by a second team member (N.D.). In other work, we have shown that these data were concordant with parent reports for highly salient health-care encounters (e.g., hospitalizations), which supports the validity of the abstraction. ${ }^{21}$ Physician visits for CAUSES patients were costed using local values (see Supplementary Materials).

To identify visits that were most likely to focus on the search for a diagnosis, only the following visits were included: (1) all consultations with medical geneticists or endocrinology and metabolism specialists; (2) the first consultation with any physician at C\&W; (3) each new consultation with a neurologist, pediatrician, or general practitioner (defined, per BC's physician fee schedule, as a visit taking place six months or longer since the patient's last appointment). The accuracy of this algorithm in capturing diagnosis-focused consultations was cross-validated using self-reported data from the caregiver survey used to capture non-C\&W physician consultations. Visits on or after patients' enrollment in CAUSES were excluded from the analysis to ensure only standard diagnostic care (i.e., pre-GWS) costs were captured.

\section{Non-C\&W physician consultations}

Data on general practitioner and specialist physician visits at a location other than $\mathrm{C} \& \mathrm{~W}$ were collected using a survey completed by one caregiver of each CAUSES patient ${ }^{21}$ in two waves: approximately two weeks following enrollment (wave 1) and six months after the ES results were returned to families (wave 2). Caregivers were asked to report the number of appointments that their child had in the six months prior to survey completion with (1) their general practitioner and/or local pediatrician (these visits were grouped together in the survey instrument), (2) other specialist physicians not based at C\&W, and (3) specialists at C\&W. In addition, caregivers had the opportunity to indicate the number of physician visits that were mostly related to the search for a diagnosis, and (in wave 2) whether their child had received a diagnosis as a result of participating in CAUSES. Parent-reported data on $\mathrm{C} \& \mathrm{~W}$ appointments were used to cross-validate our criteria for classifying an EMR-abstracted C\&W visit as diagnosis-related.

\section{Indirect costs of the diagnostic trajectory}

As part of the survey, which included an adapted version of an existing instrument, ${ }^{22,23}$ the child's caregivers were also asked to report the typical out-of-pocket transportation and accommodation costs they incurred to attend appointments at $\mathrm{C} \& \mathrm{~W}$ or with physicians elsewhere. In addition, families reported use of travel assistance programs, the typical amount of time taken off work by caregivers to accompany the child, and caregivers' and partners' employment status and wages. This allowed us to estimate the travel costs and productivity loss (using the human capital approach) associated with physician visits related to the search for a diagnosis. However, families traveling to $\mathrm{C} \& \mathrm{~W}$ from remote areas of $\mathrm{BC}$ often schedule multiple appointments for a single visit over several days because significant travel is required to get to Vancouver. Therefore, we used the EMR-derived data on C\&W visits to estimate the mean number of annual diagnostic visits to $\mathrm{C} \& \mathrm{~W}$ per patient (see Supplementary Materials).

\section{Demographics}

\section{RESULTS}

The TIDE study tracked 498 patients with unexplained intellectual developmental disorder (Table S7). The mean age at enrollment was 6.4 years $(\mathrm{sd}=4.5)$, with patients having had their first diagnostic test at a mean age of 3.2 years $(\mathrm{sd}=$ 3.6). The average time elapsed between the baseline test for a patient to their last test during the study period was 4.2 years $(s d=3.6)$. Thirty-seven percent of patients were female $(184 /$ 498), $17 \%$ had severe or profound intellectual disability and $41 \%$ moderate intellectual disability, 20\% (101/498) received GWS during the study period, and slightly more than half received a confirmed or probable etiologic diagnosis.

Caregivers of 257 CAUSES patients completed the wave 1 survey, and 88 completed the wave 2 survey (Table S8). In a previous study, we showed that the demographics of CAUSES survey respondents were highly representative of the overall CAUSES cohort. ${ }^{21}$ The average age of CAUSES patients whose caregivers responded to wave 1 was 7.6 years $(\mathrm{sd}=$ $5.0)$, and $48.2 \%(124 / 257)$ were female. Of the 88 wave 2 responses, $48.9 \%(43 / 88)$ reported having received a diagnosis from ES.

\section{Breakdown and trajectory of testing costs}

TIDE patients incurred an average of C\$6401 (95\% CI C\$6048, C\$6751) in diagnostic testing costs up to the end of the study period when the cost of GWS is included, and C\$5596 (95\% CI C\$5314, C\$5869) when GWS costs are excluded (Table 1). There were substantial differences in test utilization between patients who received GWS and those who did not, with the former incurring C $\$ 12,006$ (95\% CI $\mathrm{C} \$ 11,275, \mathrm{C} \$ 12,830)$ in costs for an average of 39.9 (95\% CI $37.0,43.1)$ tests while the latter incurred an average of C $\$ 4972$ (95\% CI C\$4708, C\$5229) in costs for 27.1 (95\% CI 26.1, 28.1) tests. Utilization of cytogenetic and physiological tests (e.g., ECG) was similar across the two groups, but utilization of diagnostic tests for GWS patients was higher for all other test 


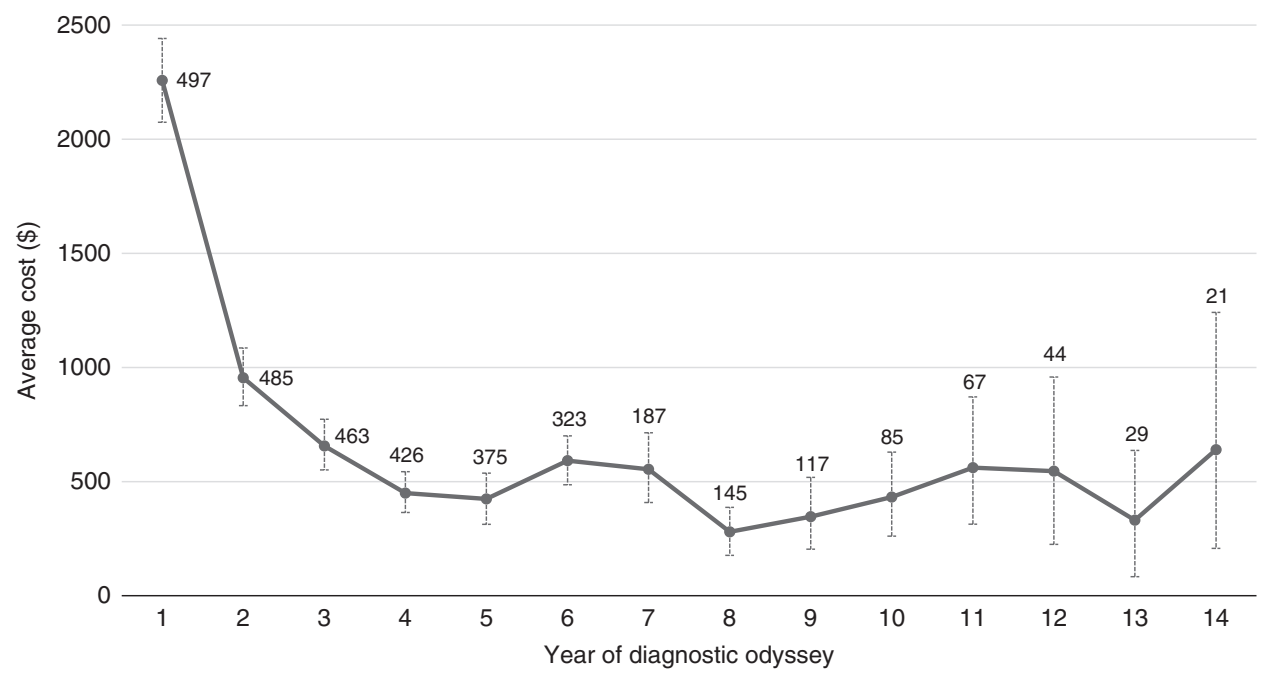

Fig. 1 Annual average per-patient non-GWS diagnostic testing costs for TIDE cohort $(\boldsymbol{n}=\mathbf{4 9 8})$. Error bars represent $95 \%$ confidence intervals. Means and $95 \%$ confidence intervals were estimated using bootstrapping. Figures above the error bars indicate the number of observations used to calculate the mean cost at each time point. GWS genome-wide sequencing.

categories. Notably, the cost of molecular genetic tests was nearly three times higher for GWS patients even when excluding the cost of GWS tests (C\$1854 vs. C $\$ 681)$. This difference is driven at least in part by the longer diagnostic trajectories experienced by GWS patients, but may also reflect clinical differences in the type of patients who received GWS.

Figure 1 shows average annual per-patient diagnostic testing costs (excluding GWS costs) in each year of patients' diagnostic odysseys (only years for which data on at least 20 patients were available are included). Average testing costs were $\mathrm{C} \$ 2257$ (95\% CI C $\$ 2074, \mathrm{C} \$ 2441$ ) in year 1, declined to C $\$ 955$ (95\% CI C $\$ 833$, C $\$ 1085$ ) in year 2, and subsequently remained stable over the remainder of the diagnostic odyssey, with an annual per-patient average of $\$ 441$ (95\% CI $\$ 401$, $\$ 486)$ in years 3 to 14 . Notably, the pattern is the same for patients who received GWS $(n=101)$ and those who did not receive GWS $(n=397)$ during the study period (Fig. S1), though GWS patient costs are consistently higher, possibly reflecting the more challenging nature of diagnosing these patients.

\section{Diagnosis-related physician consultations at C\&W}

Based on data from 91 CAUSES patients' medical records, the mean length between CAUSES patients' first consultation at $\mathrm{C} \& \mathrm{~W}$ and their enrollment in CAUSES was 4.5 years $(\mathrm{sd}=$ $2.2)$, during which they had an average of $23.6(\mathrm{sd}=26.4)$ physician consultations at $\mathrm{C} \& \mathrm{~W}$, including an average of 11.3 $(\mathrm{sd}=9.5)$ diagnosis-related consultations. Overall, $48 \%$ of $\mathrm{C} \& \mathrm{~W}$ consultations were coded as diagnosis-related, which is consistent with data from the caregiver survey. Specifically, caregivers $(n=257)$ reported an average of $2.4(\mathrm{sd}=3.0)$ $\mathrm{C} \& \mathrm{~W}$ visits for their child in the six months prior to survey admission, and an average of $1.3(\mathrm{sd}=2.1)$ diagnosis-related visits, indicating that $54 \%$ of $\mathrm{C} \& \mathrm{~W}$ visits were primarily diagnosis-related, which is in the same range as the $48 \%$ resulting from our classification criteria.
These C\&W consultations translated to an average cost up to the end of the study period of $\mathrm{C} \$ 1940$ (sd = C $\$ 1641)$ for diagnosis-related physician consultations and $\mathrm{C} \$ 2744$ (sd $=\mathrm{C}$ \$2708) for total physician consultations at C\&W for this sample of patients. The trajectory of average annual perpatient diagnosis-related physician consultation costs was similar to that for diagnostic testing costs, with a high initial consultation cost of C $\$ 631$ (95\% CI C $\$ 543, C \$ 727)$ in year 1 and a relatively stable average annual consultation cost of $\mathrm{C}$ $\$ 334$ (95\% CI C $\$ 295, \mathrm{C} \$ 369)$ per patient per year in the following seven years of patients' pre-CAUSES diagnostic trajectory (Fig. 2).

\section{Non-C\&W physician consultations}

Caregivers of CAUSES patients reported a mean of 3.3 (95\% CI $2.8,3.9$ ) physician visits outside of $\mathrm{C} \& \mathrm{~W}$ for their child in the six months prior to wave 1 survey completion, with 1.3 (95\% CI 1.0, 1.6) of those visits being primarily diagnosisrelated. This translates into an average cost of $\$ 81$ per patient every 6 months (\$162 per year) in diagnosis-related non$\mathrm{C} \& \mathrm{~W}$ physician visits. There were no statistically significant differences in utilization between families who reported a diagnosis via CAUSES and those who reported no diagnosis (Table 2$)$. The only statistically significant difference $(p<$ 0.05 ) found was between wave 1 (before GWS results) and wave 2 (6 months after return of GWS results) diagnostic physician visits for patients who received a diagnosis, which declined from an average of $1.5(95 \%$ CI $0.9,2.3)$ visits (C $\$ 93$ [95\% CI C $\$ 53, \mathrm{C} \$ 145])$ in the wave 1 survey to 0.4 (0.2 to 0.6$)$ in wave $2(\mathrm{C} \$ 22[95 \% \mathrm{CI} C \$ 12, \mathrm{C} \$ 34])$. The number of diagnostic visits remained stable for those who did not receive a diagnosis (Table 2).

\section{Indirect costs}

Average indirect costs per family per visit to $\mathrm{C} \& \mathrm{~W}$ were $\mathrm{C}$ $\$ 593$ (95\% CI: C $\$ 495, C \$ 708)$, with individual means of C $\$ 48$ 


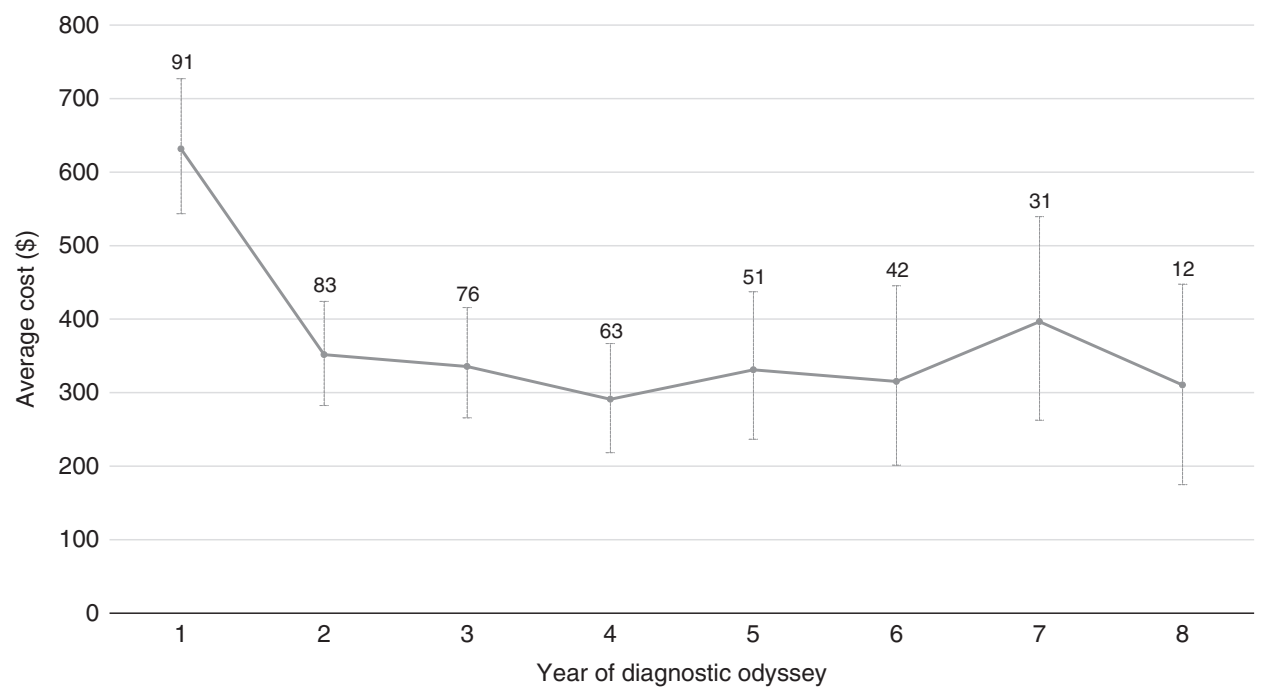

Fig. 2 Annual average per-patient diagnostic consultation costs at British Columbia Children's and Women's Hospitals (C\&W) for Clinical Assessment of the Utility of Sequencing and Evaluation as a Service study (CAUSES) patients $(\boldsymbol{n}=\mathbf{9 1})$. Error bars represent $95 \%$ confidence intervals. Means and 95\% confidence intervals were estimated using bootstrapping. Figures above the error bars indicate the number of observations used to calculate the mean cost at each time point.

(95\% CI C $\$ 33, \mathrm{C} \$ 62)$ for accommodation, C\$182 (95\% CI $\mathrm{C} \$ 143, \mathrm{C} \$ 227)$ for transportation, and C $\$ 363$ (95\% CI C $\$ 301$, $\mathrm{C} \$ 446)$ for productivity loss (Table 3 ). Average transportation and productivity costs associated with visits to patients' family doctor/local pediatrician and other non-C\&W specialists totaled C $\$ 111$ (95\% CI C $\$ 94, \mathrm{C} \$ 129)$ and $\mathrm{C} \$ 321$ (95\% CI $\mathrm{C} \$ 176, \mathrm{C} \$ 502)$ per visit, respectively. The mean number of annual diagnostic visits per patient to $\mathrm{C} \& \mathrm{~W}$ estimated from the CAUSES EMR data ranged from 1.9 to 3.5 (Table S6), with 3.5 (95\% CI 2.8, 4.1) visits in year 1 of the diagnostic trajectory, an average of $2.2(95 \%$ CI $2.0,2.6)$ visits in subsequent years, and an overall average of 2.5 (95\% CI 2.3, 2.8) visits per year. Given an annual mean of 1.8 (95\% CI 1.4 , 2.2) diagnostic visits to a family doctor/local pediatrician and $0.7(95 \%$ CI $0.4,1.2)$ visits to a non-C\&W specialist, this implies a total average of $\mathrm{C} \$ 1907$ per family per year in indirect costs relating to diagnostic physician consultations among CAUSES patients.

\section{DISCUSSION}

Our results provide a consistent picture of the cost trajectory of the diagnostic odyssey for children with suspected genetic disorders in British Columbia. Costs peak in the first year in which families are seeking a diagnosis, including $\mathrm{C} \$ 2257$ per patient in non-GWS diagnostic testing costs and C\$631 in diagnosis-related physician consultations at C\&W. In year 2, average direct diagnostic testing costs decline to C $\$ 954$ per patient, and then to $C \$ 441 /$ patient/year in subsequent years. Similarly, direct C\&W consultation costs decline to $\mathrm{C} \$ 334$ /year after year 1. Due to funding limitations, GWS has not been routinely available clinically in British Columbia, and many patients for whom it is indicated have obtained GWS through research studies like CAUSES. However, given that direct diagnostic testing costs incurred in the first two years of the diagnostic trajectory $(\mathrm{C} \$ 3211)$ are equivalent to the cost of 7.3 subsequent years of non-GWS diagnostic testing, our findings suggest that if a health system makes the decision to offer clinical GWS to children with suspected genetic disorders, the intervention is likely to avert the greatest amount of other diagnostic costs if delivered in the first two years after genetic disease is first suspected, rather than in later years.

Indeed, a set of recent studies evaluating the costeffectiveness of ES for infants and children with suspected Mendelian diseases has found that using ES as a first-line test ${ }^{11-13}$ and reanalysis of the ES data at 18 months ${ }^{14}$ may be cost-saving. However, these evaluations used time horizons equivalent to the period for which patient data were available, which ranged from $1.1^{11}$ to $6^{12}$ years. Notably, an expanded evaluation of the use of ES in infants ${ }^{11,14}$ did not find it to be cost-saving ${ }^{24}$ because it evaluated an early-ES model in which ES replaced most genetic and biochemical tests, but was not a first-line test. While this evaluation used a longer time horizon (20 years), diagnostic costs were assumed to be limited to those observed during the combined study periods $\left(2.4\right.$ years $\left.^{11,14}\right)$. Our second notable finding-the stability of costs over time for patients seeking a diagnosis-suggests that this may not be warranted because families of undiagnosed patients will continue to pursue new diagnostic investigations over long periods of time. Figs. 1 and 2 show that, at least for patients whose caregivers and physicians remain engaged in the search for a diagnosis, the cost of diagnostic care appears to persist indefinitely at a lower but stable level. If ongoing diagnostic costs for undiagnosed patients had been projected over the 20-year time horizon (or at least a larger portion thereof) in Schofield et al., ${ }^{24}$ the estimated cost-effectiveness of ES would likely have improved. This example illustrates the 


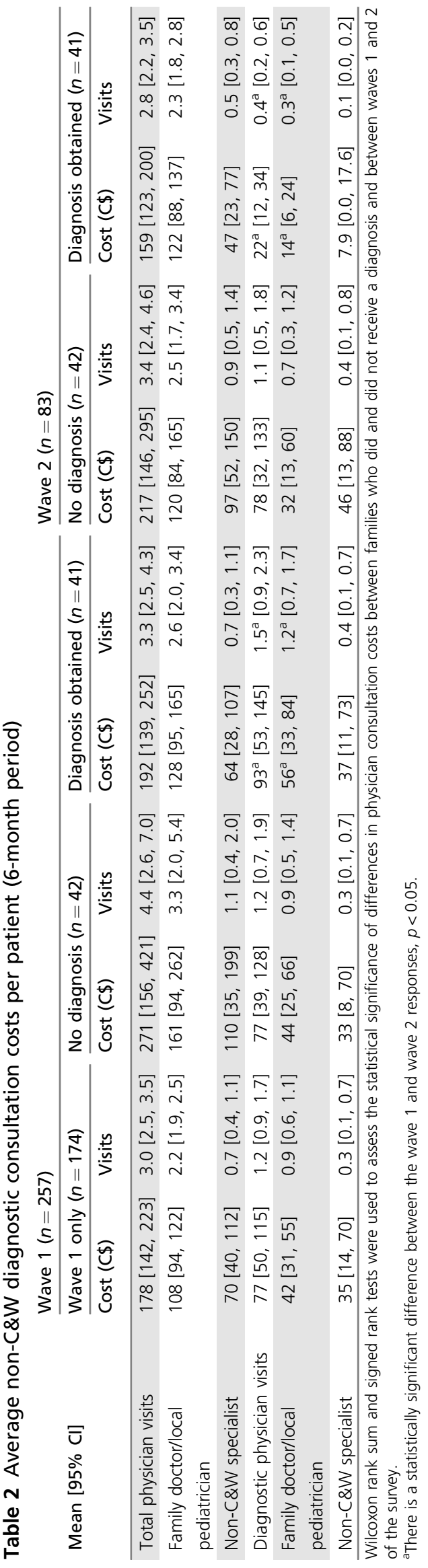

value of the long-term real-world data we present in this study.

A key open question is how long diagnostic costs continue to accrue at the same rate following a negative GWS test, given mixed evidence about whether diagnostic testing costs decline following uninformative ES. ${ }^{14,25,26}$ Our survey-based findings suggest that, at a minimum, diagnosis-related physician consultations outside of C\&W did not decline for patients who did not obtain a diagnosis through CAUSES. If diagnostic care declines substantially following uninformative GWS (possibly because families and/or physicians perceive it to have been a last resort test), this would increase the probability that GWS is cost-saving, and is therefore an important area for future research.

It is also important to note that the patients enrolled in TIDE and CAUSES were, by design, patients whose caregivers continued to seek a diagnosis. As a result, this group may not be entirely representative of the broader set of undiagnosed patients who began their diagnostic odyssey in the same period, because some families may have given up on obtaining a diagnosis prior to the beginning of the studies. In addition, these cohorts do not include patients with genetic disorders that were easily diagnosed-e.g., those with Down syndrome. The CAUSES patients were by design patients who had not received a diagnosis as a result of standard diagnostic care, so the physician consultation cost estimates may not be fully representative of the broader patient population. While the TIDE-BC cohort included patients diagnosed through standard care, the patients were selected in part based on indications of possible metabolic disease, so it is possible that utilization patterns in one cohort would not apply to the other. However, the similarity in the trajectory of testing costs for TIDE patients who did receive GWS - many of whom did so through the CAUSES study-and those who did not (Fig. S1) suggests that these cohorts were in fact comparable.

Another limitation is the reliability of caregiver-reported health-care resource utilization estimates. In a separate study, we found that CAUSES caregivers reliably reported highsalience medical encounters like hospitalizations, but tended to underreport routine encounters like physician visits ( 20\% of patients with at least one visit reported no visits). ${ }^{21}$ Our estimates of diagnosis-related physician visits outside of C\&W may therefore underestimate the true cost of diagnostic care, though the potential effect on the overall cost estimate would likely be relatively small given the larger testing and C\&W consultation costs. Moreover, while it is possible that survey nonresponse and attrition bias may reduce the generalizability of our survey-based estimates to the overall CAUSES cohort, in previous work we have shown that survey responders were broadly representative of the CAUSES cohort, ${ }^{21}$ and Table S8 does not reveal any statistically significant demographic differences between respondents to waves 1 and 2 of the survey. That said, no measures of severity of illness were available, and it is possible that families with the most severely affected children are underrepresented, so our health-care utilization estimates may be conservative. Finally, it is 
Table 3 Indirect costs per family per visit (W1 survey)

\begin{tabular}{|c|c|c|c|}
\hline Mean $(95 \% \mathrm{Cl})$ & C\&W visit $(n=195)$ & Non-C\&W specialist $(n=65)$ & Local GP/pediatrician $(n=167)$ \\
\hline Accommodation cost (C $\$$ ) & $48[33,62]$ & - & - \\
\hline Transportation cost (C\$) & $182[143,227]$ & $55[28,93]$ & $21[15,28]$ \\
\hline Productivity loss (hours) & $11.3[9.5,13.4]$ & $7.5[4.9,10.7]$ & $3.1[2.7,3.6]$ \\
\hline Productivity loss (C\$) & $363[301,446]$ & $266[147,420]$ & $91[76,106]$ \\
\hline Total indirect cost (C\$) & $593[495,708]$ & $321[176,502]$ & $111[94,129]$ \\
\hline
\end{tabular}

Cl confidence interval, C\&W British Columbia Children's and Women's Hospitals, GP general practitioner. Bolded row shows total indirect costs.

important to note that while we costed GWS tests based on the laboratory prices to maintain comparability with other tests, in a clinical setting services like genetic counseling, the increased time required for variant interpretation by specialist physicians, and the possibility of periodic reanalysis of results can all increase the overall cost of delivering a GWS service ${ }^{5}$ and should be accounted for in economic evaluations.

The costs we report are specific to British Columbia, but the shape of the diagnostic trajectory is likely to be relevant for other jurisdictions. Specifically, our results suggest that after an initial spike in testing, the average annual per-patient cost of seeking a diagnosis remains relatively stable for many patients with a suspected, but undiagnosed, genetic disease. As a result, researchers who are evaluating specific clinical implementations of pediatric diagnostic GWS programs may find that it is sufficient to capture only $2-3$ years of utilization data to estimate future costs of diagnostic investigations for patients who do not receive a diagnosis through GWS. Our results also point to the need to use longer time horizons when developing economic models for diagnostic GWS services, since accounting for the long-term ongoing diagnosis-related costs for undiagnosed patients may substantially change conclusions about the cost-effectiveness of offering diagnostic GWS.

\section{SUPPLEMENTARY INFORMATION}

The online version of this article (https://doi.org/10.1038/s41436019-0635-6) contains supplementary material, which is available to authorized users.

\section{ACKNOWLEDGEMENTS}

This study was supported by grants from the BC Children's Hospital Foundation ( $1^{\text {st }}$ Collaborative Area of Innovation to TIDEBC), the Rare Diseases Foundation (C.D.M.v.K.), Rare Disease Foundation Microgrant (P.B. and D.G.), the Canadian Institutes of Health Research (CIHR) grant 301221 (to C.D.M.v.K), and the CIHR New Emerging Team for Rare Diseases grant (to L.D.L., funding reference number 119193), as well as by the CAUSES Clinic study (J.M.F.). The CAUSES Clinic is made possible by a $C \$ 3$ million donation from Mining for Miracles through BC Children's Hospital Foundation and is supported by Genome British Columbia, the Provincial Health Services Authority, BC Children's and Women's Hospitals, the Provincial Medical Genetics Program, BC Children's Hospital Pathology and Laboratory Medicine, and the University of British Columbia. C.D.M.v.K. is recipient of the
Michael Smith Foundation for Health Research Scholar Award and a Stichting Metakids salary stipend.

\section{DISCLOSURE}

The authors declare no conflicts of interest.

Publisher's note: Springer Nature remains neutral with regard to jurisdictional claims in published maps and institutional affiliations.

\section{REFERENCES}

1. Smith HS, Swint JM, Lalani SR, et al. Clinical application of genome and exome sequencing as a diagnostic tool for pediatric patients: a scoping review of the literature. Genet Med. 2019;21:3-16.

2. Clark MM, Stark Z, Farnaes L, et al. Meta-analysis of the diagnostic and clinical utility of genome and exome sequencing and chromosomal microarray in children with suspected genetic diseases. NPJ Genomic Med. 2018:3:16.

3. Wright CF, FitzPatrick DR, Firth HV. Paediatric genomics: diagnosing rare disease in children. Nat Rev Genet. 2018;19:253-268.

4. Wright CF, Fitzgerald TW, Jones WD, et al. Genetic diagnosis of developmental disorders in the DDD study: a scalable analysis of genomewide research data. Lancet. 2015;385:1305-1314.

5. Dragojlovic N, Elliott AM, Adam S, et al. The cost and diagnostic yield of exome sequencing for children with suspected genetic disorders: a benchmarking study. Genet Med. 2018;20:1013-1021.

6. Schwarze K, Buchanan J, Taylor JC, Wordsworth S. Are whole-exome and whole-genome sequencing approaches cost-effective? A systematic review of the literature. Genet Med. 2018;20:1122-1130.

7. Monroe GR, Frederix GW, Salveberg SMC, et al. Effectiveness of wholeexome sequencing and costs of the traditional diagnostic trajectory in children with intellectual disability. Genet Med. 2016;18:949-956.

8. van Nimwegen KJM, Schieving JH, Willemsen MAAP, et al. The diagnostic pathway in complex pediatric neurology: a cost analysis. Eur J Pediatr Neurol. 2015;19:233-239.

9. Shashi V, McConkie-Rosell A, Rosell B, et al. The utility of the traditional medical genetics diagnostic evaluation in the context of next-generation sequencing for undiagnosed genetic disorders. Genet Med. 2014;16:176-182.

10. Soden S, Saunders CJ, Willig LK, et al. Effectiveness of exome and genome sequencing guided by acuity of illness for diagnosis of neurodevelopmental disorders. Sci Transl Med. 2014;6:1-14.

11. Stark Z, Schofield D, Alam K, et al. Prospective comparison of the costeffectiveness of clinical whole-exome sequencing with that of usual care overwhelmingly supports early use and reimbursement. Genet Med. 2017; 19:867-874

12. Tan TY, Dillon OJ, Stark $Z$, et al. Diagnostic impact and cost-effectiveness of whole-exome sequencing for ambulant children with suspected monogenic conditions. JAMA Pediatr. 2017;171:855-862.

13. Vissers LELM, van Nimwegen KJM, Schieving JH, et al. A clinical utility study of exome sequencing versus conventional genetic testing in pediatric neurology. Genet Med. 2017;19:1055-1063.

14. Stark Z, Schofield D, Martyn M, et al. Does genomic sequencing early in the diagnostic trajectory make a difference? A follow-up study of clinical outcomes and cost-effectivenes. Genet Med. 2019;21:173-180.

15. van Karnebeek CDM, Shevell M, Zschocke J, et al. The metabolic evaluation of the child with an intellectual developmental disorder: 
Diagnostic algorithm for identification of treatable causes and new digital resource. Mol Genet Metab. 2014;111:428-438.

16. Sayson B, Popurs MAM, Lafek $M$, et al. Retrospective analysis supports algorithm as efficient diagnostic approach to treatable intellectual developmental disabilities. Mol Genet Metab. 2015;115:1-9.

17. Tarailo-Graovac M, Shyr C, Ross CJ, et al. Exome sequencing and the management of neurometabolic disorders. $\mathrm{N}$ Engl J Med. 2016:374:2246-2255.

18. Harris PA, Taylor $R$, Thielke $R$, et al. Research electronic data capture (REDCap) - a metadata-driven methodology and workflow process for providing translational research informatics support. J Biomed Inform. 2009:42:377-381.

19. Jacobs $P$, Budden A, Lee KM. Guidance Document for the Costing of Health Care Resources in the Canadian Setting, Second Edition. Canadian Agency for Drugs and Technologies in Health (CADTH), Ottawa, Ontario, Canada. 2016.

20. Elliott AM, du Souich C, Adam S, et al. The Genomic Consultation Service: a clinical service designed to improve patient selection for genome-wide sequencing in British Columbia. Mol Genet Genomic Med. 2018 May 30; https://doi.org/10.1002/mgg3.410. Epub ahead of print].

21. Dragojlovic N, Kim E, Elliott A, CAUSES Study, Friedman JM, Lynd LD. Evaluating the use of parental reports to estimate healthcare resource utilization in children with suspected genetic disorders. J Eval Clin Pract. 2018;24:416-422

22. Genereaux D, Bansback N, Birch P. Development and pilot testing of a tool to calculate parental and societal costs of raising a child with intellectual disability. J Intellect Dev Disabil. 2016;41:11-20.

23. Genereaux D, van Karnebeek CDM, Birch PH. Costs of caring for children with an intellectual developmental disorder. Disabil Health J. 2015;8:646-651.

24. Schofield D, Rynehart L, Shresthra R, et al. Long-term economic impacts of exome sequencing for suspected monogenic disorders: diagnosis, management, and reproductive outcomes. Genet Med. 2019 May 21; https://doi.org/10.1038/s41436-019-0534-x. [Epub ahead of print].

25. Vrijenhoek T, Middleburg EM, Monroe GR, et al. Whole-exome sequencing in intellectual disability; cost before and after a diagnosis. Eur J Hum Genet. 2018. [Epub 29 June 2018].

26. Matias M, Wusik K, Neilson D, Zhang X, Valencia CA, and Collins $K$. Comparison of medical management and genetic counselling options pre- and post-whole exam sequencing for patients with positive and negative results. J Genet Couns. 2019;28:182-193.

\section{CAUSES Study}

Shelin Adam ${ }^{3,5}$, Nick Dragojlovic ${ }^{1}$, Christèle du Souich ${ }^{3,5}$, Alison M. Elliott ${ }^{3,5}$, Anna Lehman ${ }^{3,5}$, Larry Lynd ${ }^{1,6}$, Jill Mwenifumbo ${ }^{3,7}$, Tanya N. Nelson ${ }^{3,5,8,9}$, Clara van Karnebeek ${ }^{3,4,7}$ and Jan M. Friedman ${ }^{3,5}$

${ }^{7}$ Centre for Molecular Medicine and Therapeutics, University of British Columbia, Vancouver, BC, Canada. ${ }^{8}$ Department of Pathology and Laboratory Medicine, Faculty of Medicine, University of British Columbia, Vancouver, BC, Canada. ${ }^{9}$ Department of Pathology and Laboratory Medicine, BC Children's Hospital, Vancouver, BC, Canada 